Original paper

\title{
Tungsten-based material as promising new lead-free gamma radiation shielding material in nuclear medicine
}

\author{
Nadin Jamal AbuAlRoos ${ }^{\mathrm{a}}$, Mira Natasha Azman ${ }^{\mathrm{a}}$, Noorfatin Aida Baharul Amin ${ }^{\mathrm{a}}$, \\ Rafidah Zainon ${ }^{\mathrm{a}, \mathrm{b}, *}$ \\ a Oncological and Radiological Sciences Cluster, Advanced Medical and Dental Institute, SAINS@BERTAM, Universiti Sains Malaysia, 13200 Kepala Batas, Pulau Pinang, \\ Malaysia \\ ${ }^{\mathrm{b}}$ School of Physics, Universiti Sains Malaysia, 11800 Gelugor, Pulau Pinang, Malaysia
}

\section{A R T I C L E I N F O}

\section{Keywords:}

Attenuation coefficient

Nuclear medicine

Radiation shielding materials

Tungsten carbide

\begin{abstract}
A B S T R A C T
Purpose: The main objective of this study was to evaluate the efficacy of tungsten carbide as new lead-free radiation shielding material in nuclear medicine by evaluating the attenuation properties.

Materials and methods: The elemental composition of tungsten carbide was analysed using Field-Emission Scanning Electron Microscopy (FESEM) with energy dispersive X-ray (EDX). The purity of tungsten carbide was 99.9\%, APS: 40-50 $\mu \mathrm{m}$. Three discs of tungsten carbide was fabricated with thickness of $0.1 \mathrm{~cm}, 0.5 \mathrm{~cm}$ and $1.0 \mathrm{~cm}$. Three lead discs with similar thickness were used to compare the attenuation properties with tungsten carbide discs. Energy calibration of gamma spectroscopy was performed by using ${ }^{123} \mathrm{I},{ }^{133} \mathrm{Ba},{ }^{152} \mathrm{Eu}$, and ${ }^{137} \mathrm{Cs}$. Gamma radiation from these sources were irradiated on both materials at energies ranging from $0.160 \mathrm{MeV}$ to $0.779 \mathrm{MeV}$. The experimental attenuation coefficients of lead and tungsten carbide were compared with theoretical attenuation coefficients of both materials from NIST database. The half value layer and mean free path of both materials were also evaluated in this study.

Results: This study found that the peaks obtained from gamma spectroscopy have linear relationship with all energies used in this study. The relative differences between the measured and theoretical mass attenuation coefficients are within $0.19-5.11 \%$ for both materials. Tungsten carbide has low half value layer and mean free path compared to lead for all thickness at different energies.

Conclusion: This study shows that tungsten carbide has high potential to replace lead as new lead-free radiation shielding material in nuclear medicine.
\end{abstract}

\section{Introduction}

Lead has been used as radiation shielding material in various applications including diagnostic imaging, radiation therapy, nuclear and industrial shielding. Lead is one of the radiation shielding materials that commonly used for X-rays and gamma radiation because it has high density, high atomic number, and high linear and mass attenuation coefficients [1-4]. Lead can be hazardous when taken into the body by swallowing or breathing in lead or materials contaminated with lead. Radiation workers sometimes bring lead residues into their home on their work clothes, skin, hair and equipment after dealing with lead at their workplace [5-7].

Gamma ray is penetrating electromagnetic radiation that can damage living cells by transferring its energy to surrounding cells. Gamma rays are high-energy photons with very short wavelengths and thus, very high frequency [8]. Gamma rays can travel thousands of feet in air and it can easily pass through the human body. The attenuation of gamma radiation occurs through the interaction of the gamma radiation with matter. The degree of gamma ray attenuation depends on the energy of incident gamma radiation, atomic number, density of elements in the shielding material and the thickness of shielding [9].

Thus, appropriate radiation shielding must be applied to protect human and the environment from the harmful effects of radiation. In medical application, radiation dose to workers is reduced as low as possible by applying appropriate radiation shielding.

High atomic number of elements play an important role in radiation protection. This is due to probability of occurrence of photoelectric absorption per atom within a particular material depends on the energy of incident X-ray photons and the atomic number of atoms comprising the irradiated object. The photoelectric absorption increases markedly

\footnotetext{
* Corresponding author.

E-mail address: rafidahzainon@usm.my (R. Zainon).
} 
as the energy of the incident photon decreases and the atomic number of the irradiated atoms increases [10]. Choosing the most appropriate type of radiation shielding is mainly based on the cost, weight, chemical and physical durability of the materials. High density and atomic number materials offer superior attenuation to low atomic number materials.

The use of toxic heavy metal like lead causes serious long-term effects on health and environment. Thus, it motivates interests in finding non-toxic, light, flexible and low-cost radiation shielding materials to replace lead. There is an urgent needs to replace lead radiation shielding material for radiation protection $[11,12]$.

The use of lead has been restricted in many equipment and applications according to RoHS (Restriction of Hazardous Substances) directive of European union [13]. In 2002 and 2011 (and recast it in 2019), European Union accepted a directive on the restriction of use of lead in electrical and electronic equipment. However, this restriction does not involve using lead in nuclear and medical equipment $[14,15]$.

Lead free material such as tungsten is non-toxic material. In addition, tungsten has high density and better shielding properties compared to lead due to its higher atomic number [16]. However, pure tungsten is very expensive. Pure tungsten has higher attenuation properties and smaller half value layer half value layer compared to conventional lead shielding material $[17,18]$.

A study on ultra-high molecular weight polyethylene with addition of tungsten powder was performed and it showed that ultra-high molecular weight polyethylene is one of the polymers resistant to ionising radiation [19]. The presence of tungsten particles in the ultra-high molecular polyethylene structure enhanced its shielding properties. Attenuation coefficient increases with increasing tungsten content in the composite [20].

The radiation shielding properties of tungsten and epoxy composite were investigated for low energy gamma rays at $122 \mathrm{keV}$ [21]. Results showed that high percentage of tungsten increase radiation shielding properties of the sample. This study showed that tungsten is a promising material to replace conventional lead material because it has non-toxic properties and it has high efficiency in neutron radiation attenuation [222324]. Moreover, Soylu et al., study showed that radiation shielding efficiency of tungsten carbide and ethylene vinyl acetate polymer composite were similar to lead at $364 \mathrm{keV}$ and $662 \mathrm{keV}$ gamma photon energies [25].

Therefore, there is there is an urgent need to replace the conventional lead-free material for radiation protection. This study focuses on the evaluation of the efficacy of tungsten carbide as new lead-free radiation shielding material in nuclear medicine by analysing the attenuation properties.

\section{Materials and methods}

\subsection{Elemental composition analysis of tungsten carbide}

The Field-Emission Scanning Electron Microscopy (FESEM) with energy dispersive X-ray (EDX) model (JSM - 6460 LV) were used to analyse tungsten carbide material at Nano-Optoelectronics Research and Technology Laboratory, Universiti Sains Malaysia. The EDX was used to analyse sample purity by evaluating the elemental and chemical compounds in the sample. Purity of the compound and the homogenous distribution of the particles are important for the accuracy of measurements. Tungsten carbide powder was used in this study. It was purchased from Mayinglong Pharmaceutical Group Ltd. The purity of tungsten carbide was 99.9\%, APS: 40-50 $\mu \mathrm{m}$ (See Fig. 1).

\subsection{Preparation of tungsten carbide and lead discs.}

In this study, tungsten carbide powder was formed into several discs shape with various thickness. Fig. 2 shows manual hydraulic press machine (Specac) used in this study to prepare the materials into disc shape with various thickness. The tungsten carbide powder was placed on an evacuable pellet die and it was compressed into three different discs thickness; $0.1 \mathrm{~cm}, 0.5 \mathrm{~cm}$, and $1.0 \mathrm{~cm}$ as shown in Fig. 3. The lead discs with similar thickness of $0.1 \mathrm{~cm}, 0.5 \mathrm{~cm}$ and $1.0 \mathrm{~cm}$ were used in this study for comparative analysis of attenuation properties. Fig. 4 shows $1.0 \mathrm{~cm}$ thickness of tungsten carbide and $1.0 \mathrm{~cm}$ thickness of lead disc.

\subsection{Energy calibration and measurement of attenuation coefficient of tungsten carbide and lead discs.}

Energy calibration of gamma spectroscopy was performed by determining energies of ${ }^{123} \mathrm{I},{ }^{133} \mathrm{Ba},{ }^{152} \mathrm{Eu}$, and ${ }^{137} \mathrm{Cs}$ used in this study. The NaI detector was used to measure the gamma ray emitters. The peak distributions were measured with known sources and calibrated across the energies.

The attenuation coefficients measurement was performed by placing the tungsten carbide and lead discs between the radioactive sources and NaI detector (Fig. 5). Gamma-rays from ${ }^{123} \mathrm{I},{ }^{133} \mathrm{Ba},{ }^{152} \mathrm{Eu}$, and ${ }^{137} \mathrm{Cs}$ were irradiated on tungsten carbide and lead samples with various thickness to evaluate the attenuation coefficient properties at energies ranging from $0.160 \mathrm{MeV}$ to $0.779 \mathrm{MeV}$. Three measurement were performed for each sample thickness at each gamma energy. Fig. 5 shows the experimental set-up for tungsten carbide disc, NaI detector and radioactive source used in this study.

Gamma transmissivity were analysed to evaluate radiation shielding performance of the materials. The mass attenuation coefficient $(\mu / \rho)$ was derived from Lambert-Beer's law equation. The attenuation of gamma radiation was calculated from Eqs. (1) to (3).

$I=I_{0} e^{-\mu x}$

where $I_{o}$ denotes the initial photon intensity; $I$ is the photon intensity transmitted through sample with thickness, $x$ of the material.

Determination of mass attenuation coefficient of the materials was performed by dividing the linear attenuation coefficient of the material by the density of the material.

$\mu / \rho=x^{-1} \ln \left(I_{0} / I\right)$

where $I_{o}$ denotes the initial photon intensity; $I$ the photon intensity transmitted through sample with thickness $x$, and $\rho$ is the sample density.

The transmittance of gamma rays was defined by the output intensity relative to the input intensity as a function of sample thickness and the beam energy. The relative difference between theoretical and experimental values of mass attenuation coefficient was evaluated by $\Delta(\mu / \rho)$ which is defined as shown in Eq. (3).

$\Delta\left(\frac{\mu}{\rho}\right)=\frac{\left(\frac{\mu}{\rho}\right)_{\text {theo }}-\left(\frac{\mu}{\rho}\right)_{\exp t}}{\left(\frac{\mu}{\rho}\right)_{\text {theo }}}$

In this study, gamma-rays from ${ }^{123} \mathrm{I},{ }^{133} \mathrm{Ba},{ }^{152} \mathrm{Eu}$ and ${ }^{137} \mathrm{Cs}$ passed through the samples. A high-resolution of NaI scintillation detector was used to detect gamma radiation that penetrated through samples. The energy range chosen in this study was between $0.160 \mathrm{MeV}$ and $0.779 \mathrm{MeV}$. It is based on the energy lines of each gamma emitter used in this study.

The mass attenuation coefficients of tungsten carbide and lead were calculated in this study. Results were compared with theoretical mass attenuation coefficients of the materials obtained from XCOM simulation software [27]. Statistical analysis used in this study was two tailed paired-sample $t$-test to prove the similarity of the mass attenuation coefficients of the two materials experimentally and theoretically. The $P$-value obtained in this study was recorded to evaluate if the mass attenuation coefficients of tungsten carbide and lead have no significant difference. 


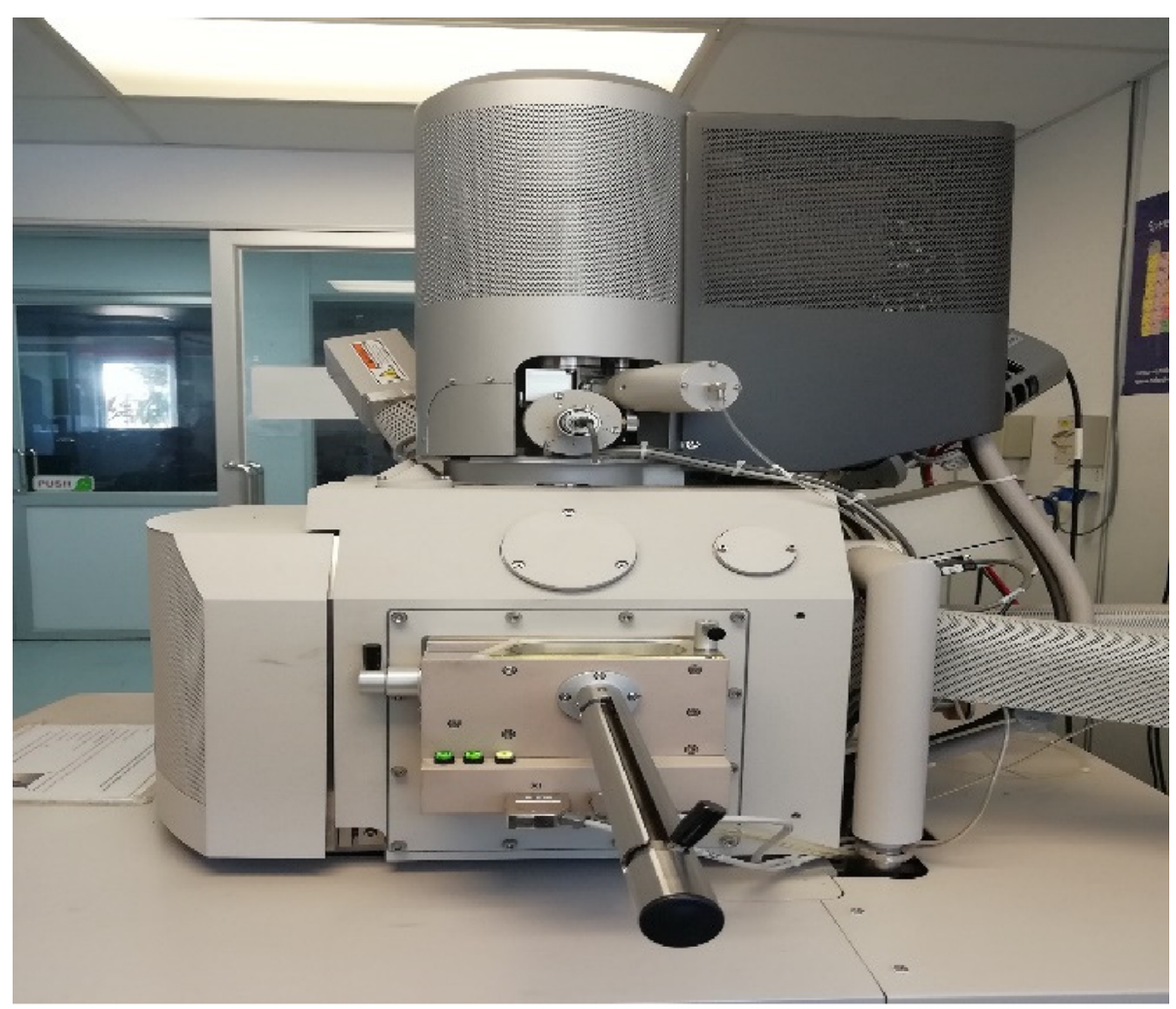

Fig. 1. The FESEM-EDX used in this study to analyse the elemental composition of tungsten carbide.

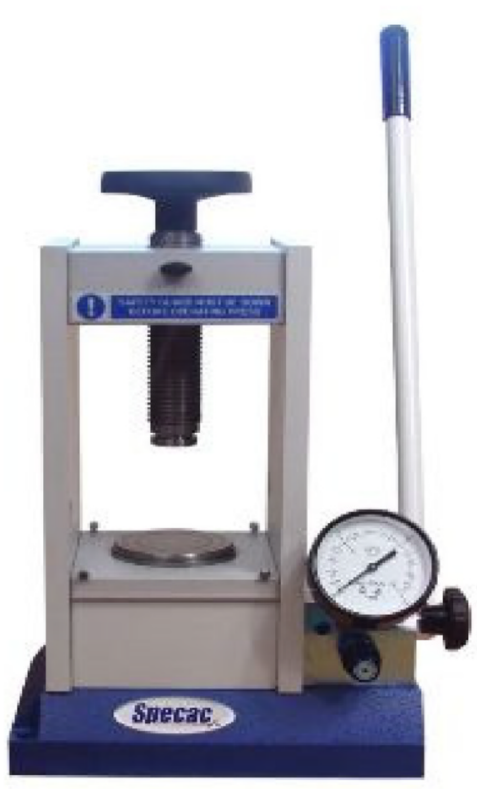

Fig. 2. Manual hydraulic press machine was used to form different disc thickness of tungsten carbide samples.

\subsection{Evaluation of half value layer and mean free path of tungsten carbide} and lead

The half value layer was evaluated in this study to analyse the penetrating ability of particular radiation through materials. It represents the thickness of an absorber that will reduce the gamma radiation to half. The HVL was evaluated by dividing $\ln 2$ with linear attenuation coefficient at specific gamma-ray energy as shown in Eq. (4).
$H V L=\frac{\ln 2}{\mu}=\frac{0.693}{\mu}$

The mean free path is the average distance of a photon travels between collisions with atoms of the target material. The mean free path of material was computed from Eq. (5).

$M F P=\frac{1}{\mu}$

\section{Results}

\subsection{Elemental composition analysis of tungsten carbide}

The elemental composition of tungsten carbide was reported in Table 1 . The atomic number of tungsten is 74 , while carbon is 6 . The density of tungsten carbide was 15.63. The detail of percentage of weight and atomic are as reported in Table 1 . There was $98.65 \%$ of tungsten and $1.35 \%$ of carbon in this study.

Fig. 6 shows elemental composition of tungsten carbide powder. The FESEM images of tungsten carbide microstructure with various magnification at $10 \mathrm{k}$ magnification, $50 \mathrm{k}$ magnification and $100 \mathrm{k}$ magnification were shown in Fig. 7.

\subsection{Results of linear and mass attenuation coefficients, half value layer and} mean free path of tungsten carbide and lead

The energy calibration of gamma spectroscopy was performed using ${ }^{123} \mathrm{I},{ }^{133} \mathrm{Ba}$, ${ }^{152} \mathrm{Eu}$, and ${ }^{137} \mathrm{Cs}$. Results were reported in Table 2 . The peaks for each radioactive sources were also reported in the table. The peak of each source at various energies was plotted in Fig. 8.

Results of linear and mass attenuation coefficients, half value layer and mean free path of tungsten carbide discs at $0.1 \mathrm{~cm}, 0.5 \mathrm{~cm}$ and $1.0 \mathrm{~cm}$ thickness were summarised in Table 3 . The average transmitted photon intensity $(I)$ and the average incident photon intensity $\left(I_{O}\right)$ were 


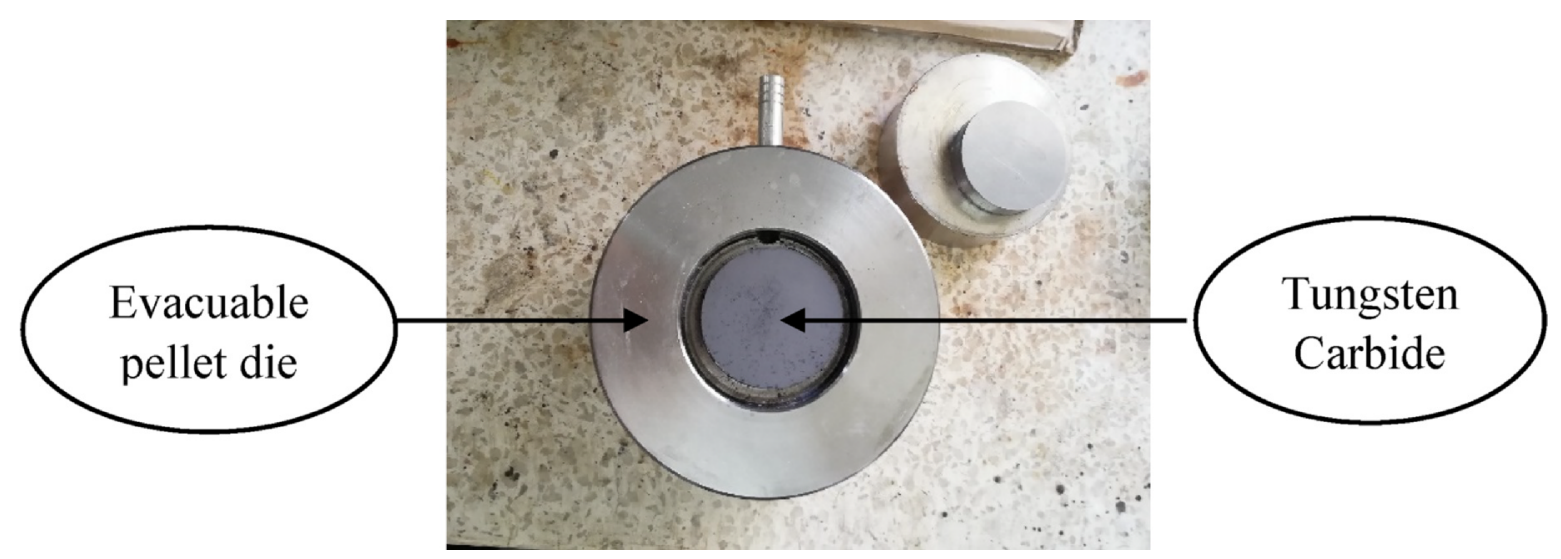

Fig. 3. Tungsten carbide powder placed on evacuable pellet die to form into disc shape.

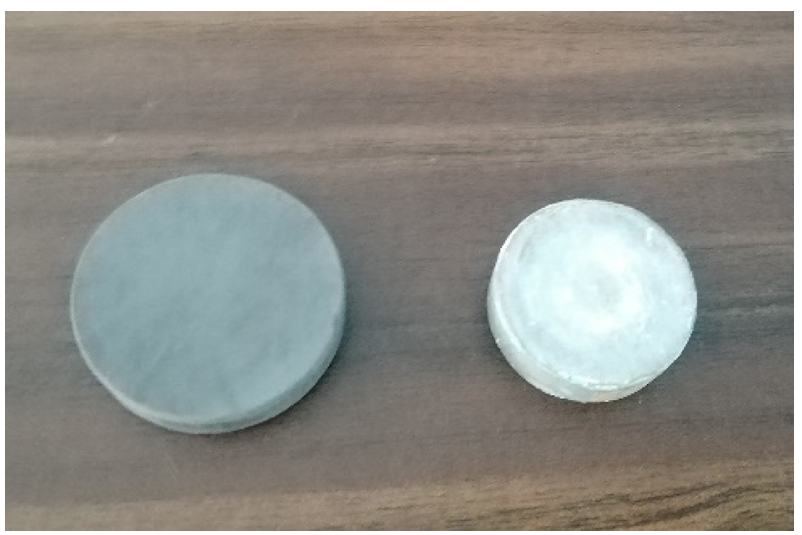

Fig. 4. The $1.0 \mathrm{~cm}$ thickness of (left) tungsten carbide disc and (right) lead disc.

also reported in the table for each energy used in this study.

In addition, Table 4 shows the linear and mass attenuation coefficients, half value layer and mean free path of lead discs at $0.1 \mathrm{~cm}$, $0.5 \mathrm{~cm}$ and $1.0 \mathrm{~cm}$ thickness. The average transmitted photon intensity $(I)$ and the average incident photon intensity $\left(I_{O}\right)$ were also summarised in the table for each energy used in this study.

The linear and mass attenuation coefficients of tungsten carbide and lead at different energies were plotted in Figs. 9 and 10 respectively. Both linear and mass attenuation coefficients were plotted for different disc thickness for all energies used in this study. The linear and mass attenuation coefficients increase when the atomic number of the absorber increases. The linear and mass attenuation coefficients for all materials decrease with the energy.

Furthermore, the half value layer and mean free path of tungsten carbide and lead were presented in Figs. 11 and 12 respectively. The half value layer and mean free path of both materials were plotted for all disc thickness at various energies. The mean free path and half value layer are higher for thicker absorber at highest energy,

In addition, the measured and theoretical mass attenuation coefficients of both tungsten carbide and lead at energies of 0.160-0.779 MeV were summarised in Table 5. Fig. 13 shows the measured and theoretical mass attenuation coefficients of tungsten carbide and lead. The relative differences between the measured and theoretical mass attenuation coefficients are within $0.19-5.11 \%$ for both lead and tungsten carbide.

Table 6 shows mass attenuation coefficient of tungsten carbide obtained from this study and compared to B. Buyuk [17] and Yingwei Hou studies [26]. It shows that tungsten carbide attenuates more radiation compared to pure tungsten, tungsten carbide-cobalt and tungsten reinforced with fiber.

\section{Discussion}

In this study, three different thickness of tungsten carbide discs were successfully fabricated for attenuation properties characterisation. The thickness of fabricated tungsten carbide discs were $0.1 \mathrm{~cm}, 0.5 \mathrm{~cm}$ and $1.0 \mathrm{~cm}$. There were three similar thickness of lead discs were used for comparative analysis of attenuation properties of both materials. The gamma ray attenuation coefficients provide valuable information on the gamma radiation shielding performance of absorber materials. The gamma spectroscopy was calibrated with known sources in this study; ${ }^{123} \mathrm{I},{ }^{133} \mathrm{Ba},{ }^{152} \mathrm{Eu}$, and ${ }^{137} \mathrm{Cs}$. It is found that the peaks obtained from gamma spectroscopy of radioactive sources have linear relationship with all energies used in this study.

The attenuation coefficient is an important parameter to investigate the interaction of radiation with matter. The attenuation coefficients of tungsten carbide and lead samples decrease with increasing photon

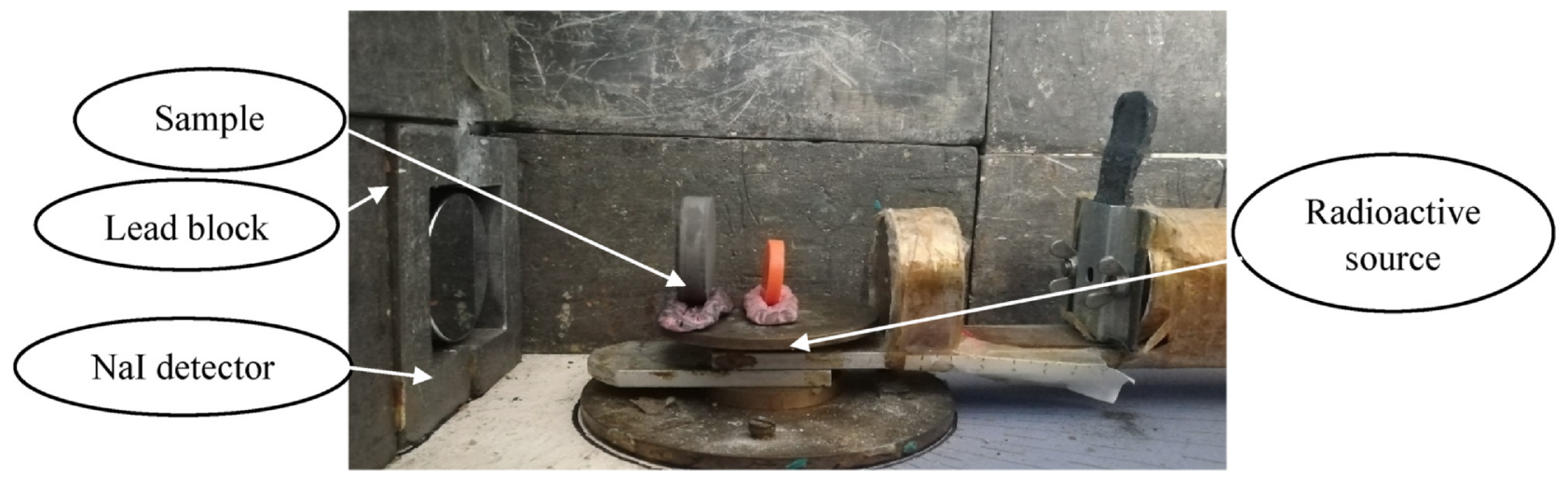

Fig. 5. The experiment set-up for measurement of attenuation coefficient of tungsten carbide and lead discs. 
Table 1

Elemental composition of tungsten carbide.

\begin{tabular}{|c|c|c|c|c|c|c|}
\hline Element & Symbol & Atomic number & Atomic mass & Weight percentage (\%) & Atomic percentage (\%) & Density $\left(\mathrm{g} / \mathrm{cm}^{3}\right)$ \\
\hline Tungsten & $\mathrm{W}$ & 74 & 183.84 & 98.65 & 82.65 & 15.63 \\
\hline Carbon & $\mathrm{C}$ & 6 & 12.0107 & 1.35 & 17.53 & \\
\hline
\end{tabular}

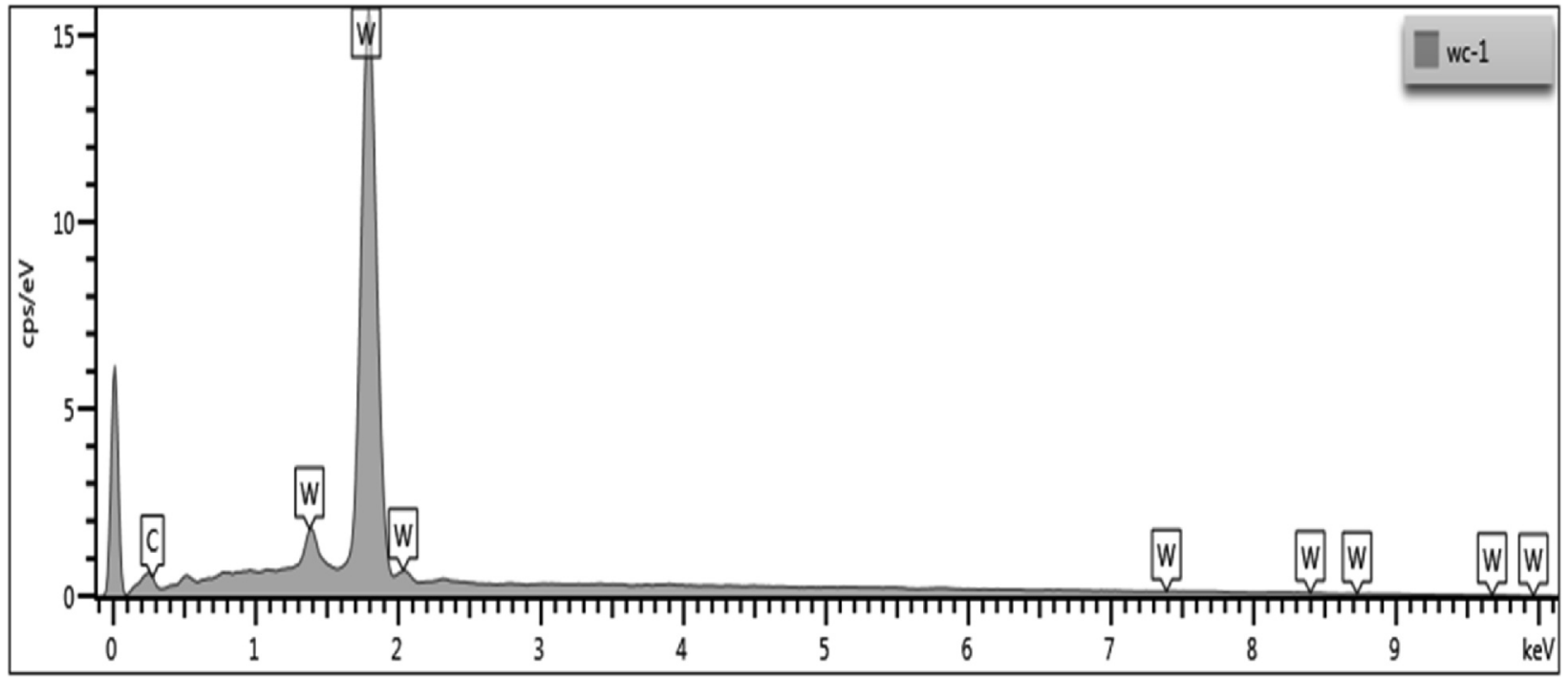

Fig. 6. The elemental composition of tungsten carbide powder.

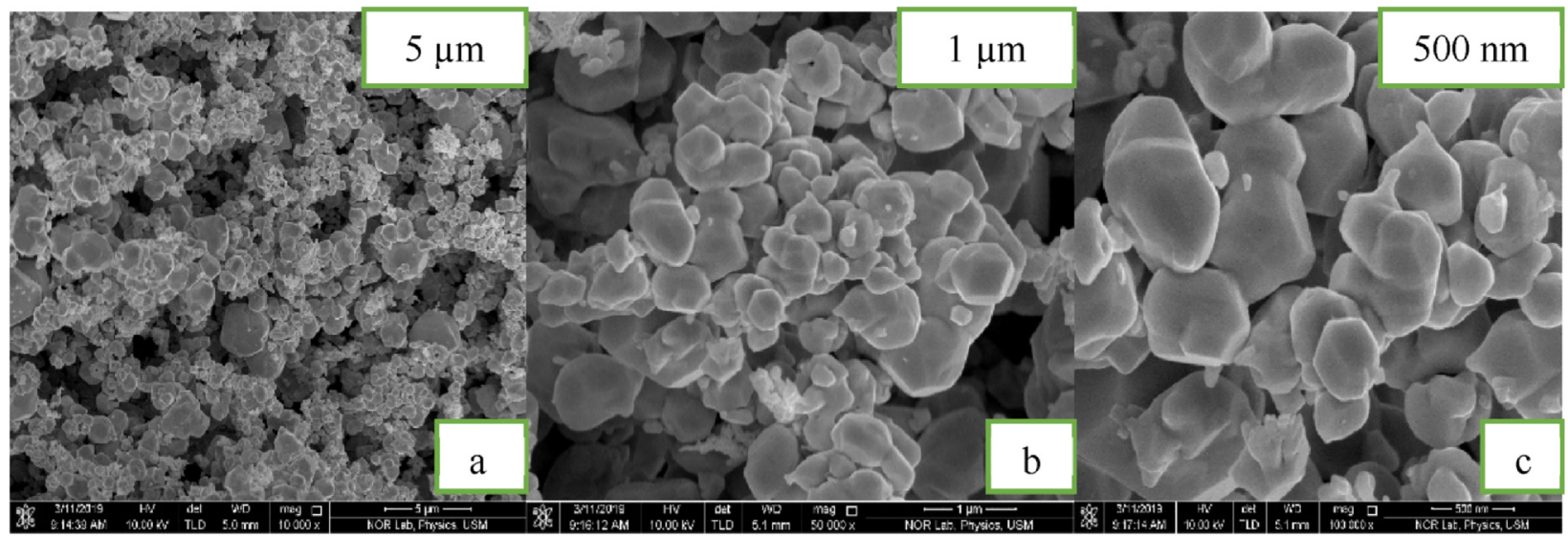

Fig. 7. The FESEM images of tungsten carbide at (a) $10 \mathrm{kx}$ magnification, (b) $50 \mathrm{kx}$ magnification and (c) $100 \mathrm{kx}$ magnification.

Table 2

The peak of each gamma emitter and the corresponding energy in MeV.

\begin{tabular}{llr}
\hline Radioactive source & Energy $(\mathrm{MeV})$ & \multicolumn{1}{c}{ Peak } \\
\hline${ }^{123} \mathrm{I}$ & 0.160 & 21.50 \\
${ }^{152} \mathrm{Eu}$ & 0.245 & 33.01 \\
${ }^{152} \mathrm{Eu}$ & 0.344 & 47.00 \\
${ }^{133} \mathrm{Ba}$ & 0.356 & 48.41 \\
${ }^{137} \mathrm{Cs}$ & 0.662 & 91.01 \\
${ }^{152} \mathrm{Eu}$ & 0.779 & 108.17 \\
\hline
\end{tabular}

energy and absorber thickness. The mass attenuation coefficients of both materials decrease with the increase of gamma ray energy. This is due to different interaction mechanism of photons at different energies. The strength of these interactions depends on the energy and the elemental composition of the material, but not much on chemical properties, since the photon energy is much higher than chemical binding energies. The photoelectric absorption dominates at low-energies, while
Compton scattering dominates at higher energies. The photoelectric process is strongly dependent on atomic number of materials and it is inversely proportional to the incident gamma energy.

On the other hand, Compton scattering process is dominant at high energy ranging from $0.60 \mathrm{Mev}$ up to several $\mathrm{MeV}$. Compton scattering process is directly proportional to physical density of material and inversely proportional to photon energy. It does not depend on atomic number of the material. Furthermore, pair production process dominates at higher energies. The probability of photon interaction via pair production process increases exponentially with the increase in photon energy [8].

This study also evaluated the measured mass attenuation coefficient of tungsten carbide and lead and compared with theoretical mass attenuation coefficients of both materials. This study found that the measured mass attenuation coefficients of tungsten carbide and lead using the gamma spectroscopy were close to the theoretical mass attenuation coefficients of both materials obtained from NIST database (XCOM). Statistical analysis results from two tailed paired-sample $t$-test 


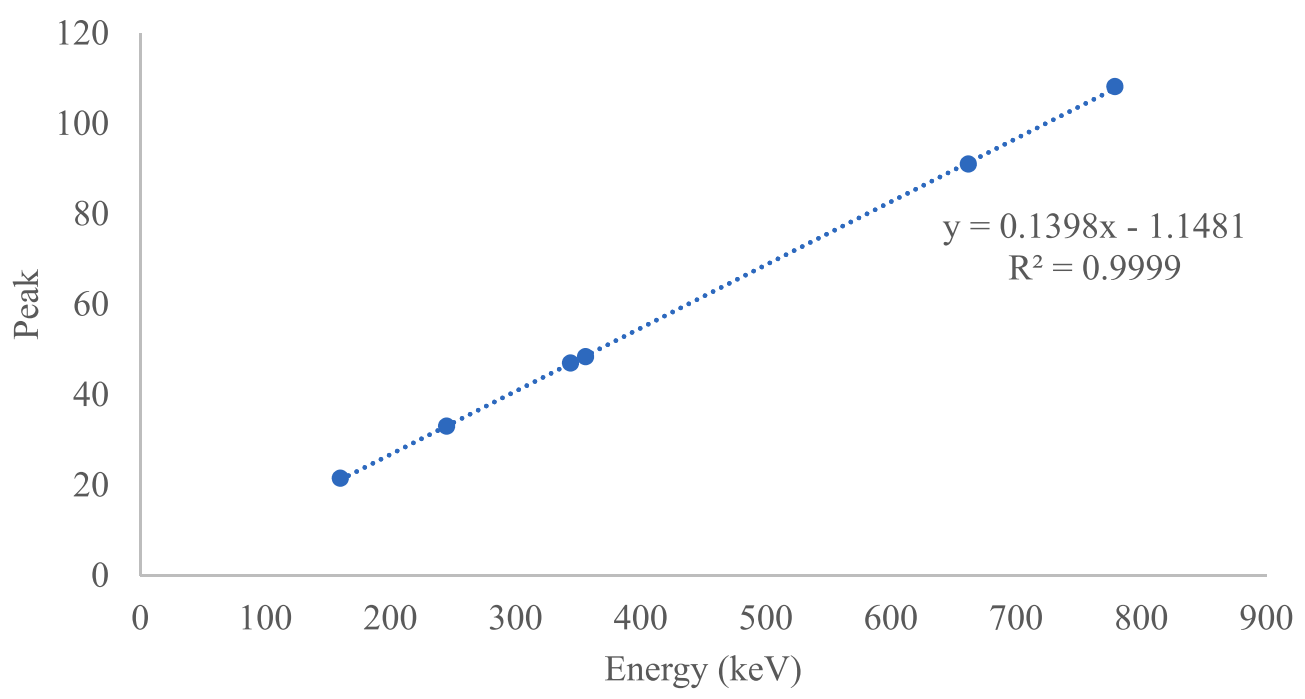

Fig. 8. The peak of each radioactive source at various energies used in this study.

Table 3

The linear and mass attenuation coefficients, half value layer and mean free path of tungsten carbide discs at $0.1 \mathrm{~cm}, 0.5 \mathrm{~cm}$ and $1.0 \mathrm{~cm}$ thickness.

\begin{tabular}{|c|c|c|c|c|c|c|c|}
\hline Thickness (cm) & $\begin{array}{l}\text { Energy } \\
(\mathrm{MeV})\end{array}$ & $\begin{array}{l}\text { The average transmitted } \\
\text { photon intensity }(I)\end{array}$ & $\begin{array}{l}\text { The average incident } \\
\text { photon intensity }\left(I_{O}\right)\end{array}$ & $\begin{array}{l}\text { Linear attenuation } \\
\text { coefficient }\left(\mathrm{cm}^{-1}\right)\end{array}$ & $\begin{array}{l}\text { Mass attenuation } \\
\text { coefficient }\left(\mathrm{cm}^{2} / \mathrm{g}\right)\end{array}$ & $\begin{array}{l}\text { Half value } \\
\text { layer }(\mathrm{cm})\end{array}$ & $\begin{array}{l}\text { Mean free } \\
\text { path }(\mathrm{cm})\end{array}$ \\
\hline \multirow[t]{6}{*}{0.1} & 0.160 & 110.00 & 685.11 & 18.29 & 1.17 & 0.03 & 0.05 \\
\hline & 0.245 & 177.42 & 341.38 & 6.54 & 0.41 & 0.10 & 0.15 \\
\hline & 0.344 & 188.69 & 308.05 & 4.90 & 0.31 & 0.14 & 0.20 \\
\hline & 0.356 & 176.30 & 277.72 & 4.54 & 0.29 & 0.15 & 0.22 \\
\hline & 0.662 & 182.29 & 245.72 & 2.98 & 0.19 & 0.23 & 0.33 \\
\hline & 0.779 & 87.14 & 100.00 & 1.37 & 0.08 & 0.50 & 0.72 \\
\hline \multirow[t]{6}{*}{0.5} & 0.160 & 104.36 & 685.11 & 3.76 & 0.24 & 0.18 & 0.26 \\
\hline & 0.245 & 117.32 & 341.38 & 2.13 & 0.13 & 0.32 & 0.46 \\
\hline & 0.344 & 111.32 & 308.05 & 2.03 & 0.13 & 0.34 & 0.49 \\
\hline & 0.356 & 99.97 & 277.72 & 2.04 & 0.13 & 0.33 & 0.48 \\
\hline & 0.662 & 96.87 & 245.72 & 1.86 & 0.11 & 0.37 & 0.53 \\
\hline & 0.779 & 70.00 & 100.00 & 0.71 & 0.04 & 0.97 & 1.40 \\
\hline \multirow[t]{6}{*}{1} & 0.160 & 98.38 & 685.11 & 1.94 & 0.12 & 0.35 & 0.51 \\
\hline & 0.245 & 89.89 & 341.38 & 1.33 & 0.08 & 0.51 & 0.74 \\
\hline & 0.344 & 89.66 & 308.05 & 1.23 & 0.07 & 0.56 & 0.81 \\
\hline & 0.356 & 84.41 & 277.72 & 1.19 & 0.07 & 0.58 & 0.83 \\
\hline & 0.662 & 80.33 & 245.72 & 1.11 & 0.07 & 0.61 & 0.89 \\
\hline & 0.779 & 53.00 & 100.00 & 0.63 & 0.04 & 1.09 & 1.57 \\
\hline
\end{tabular}

Table 4

Table 3 the linear and mass attenuation coefficients, half value layer and mean free path of lead discs at $0.1 \mathrm{~cm}, 0.5 \mathrm{~cm}$ and $1.0 \mathrm{~cm}$ thickness.

\begin{tabular}{|c|c|c|c|c|c|c|c|}
\hline Thickness $(\mathrm{cm})$ & $\begin{array}{l}\text { Energy } \\
(\mathrm{Mev})\end{array}$ & $\begin{array}{l}\text { The average transmitted } \\
\text { photon intensity }(I)\end{array}$ & $\begin{array}{l}\text { The average incident } \\
\text { photon intensity }\left(I_{O}\right)\end{array}$ & $\begin{array}{l}\text { Linear attenuation } \\
\text { coefficient }\left(\mathrm{cm}^{-1}\right)\end{array}$ & $\begin{array}{l}\text { Mass attenuation } \\
\text { coefficient }\left(\mathrm{cm}^{2} / \mathrm{g}\right)\end{array}$ & $\begin{array}{l}\text { Half value } \\
\text { layer }(\mathrm{cm})\end{array}$ & $\begin{array}{l}\text { Mean free } \\
\text { path }(\mathrm{cm})\end{array}$ \\
\hline \multirow[t]{6}{*}{0.1} & 0.160 & 112.11 & 685.11 & 18.10 & 1.59 & 0.03 & 0.05 \\
\hline & 0.245 & 178.76 & 341.38 & 6.46 & 0.57 & 0.10 & 0.15 \\
\hline & 0.344 & 173.19 & 308.05 & 5.75 & 0.50 & 0.12 & 0.17 \\
\hline & 0.356 & 200.13 & 277.72 & 3.27 & 0.28 & 0.21 & 0.30 \\
\hline & 0.662 & 208.15 & 245.72 & 1.65 & 0.14 & 0.41 & 0.60 \\
\hline & 0.779 & 92.09 & 100.00 & 0.823 & 0.07 & 0.84 & 1.21 \\
\hline \multirow[t]{6}{*}{0.5} & 0.160 & 111.94 & 685.11 & 3.62 & 0.31 & 0.19 & 0.27 \\
\hline & 0.245 & 119.88 & 341.38 & 2.09 & 0.18 & 0.33 & 0.47 \\
\hline & 0.344 & 108.21 & 308.05 & 2.09 & 0.18 & 0.33 & 0.47 \\
\hline & 0.356 & 100.00 & 277.72 & 2.04 & 0.18 & 0.33 & 0.48 \\
\hline & 0.662 & 88.72 & 245.72 & 2.03 & 0.17 & 0.34 & 0.49 \\
\hline & 0.779 & 68.05 & 100.00 & 0.76 & 0.06 & 0.90 & 1.29 \\
\hline \multirow[t]{6}{*}{1} & 0.160 & 80.11 & 685.11 & 2.14 & 0.18 & 0.32 & 0.46 \\
\hline & 0.245 & 88.15 & 341.38 & 1.35 & 0.11 & 0.51 & 0.73 \\
\hline & 0.344 & 100.13 & 308.05 & 1.12 & 0.09 & 0.61 & 0.88 \\
\hline & 0.356 & 98.76 & 277.72 & 1.03 & 0.09 & 0.67 & 0.96 \\
\hline & 0.662 & 88.19 & 245.72 & 1.02 & 0.09 & 0.67 & 0.97 \\
\hline & 0.779 & 52.09 & 100.00 & 0.65 & 0.05 & 1.06 & 1.53 \\
\hline
\end{tabular}




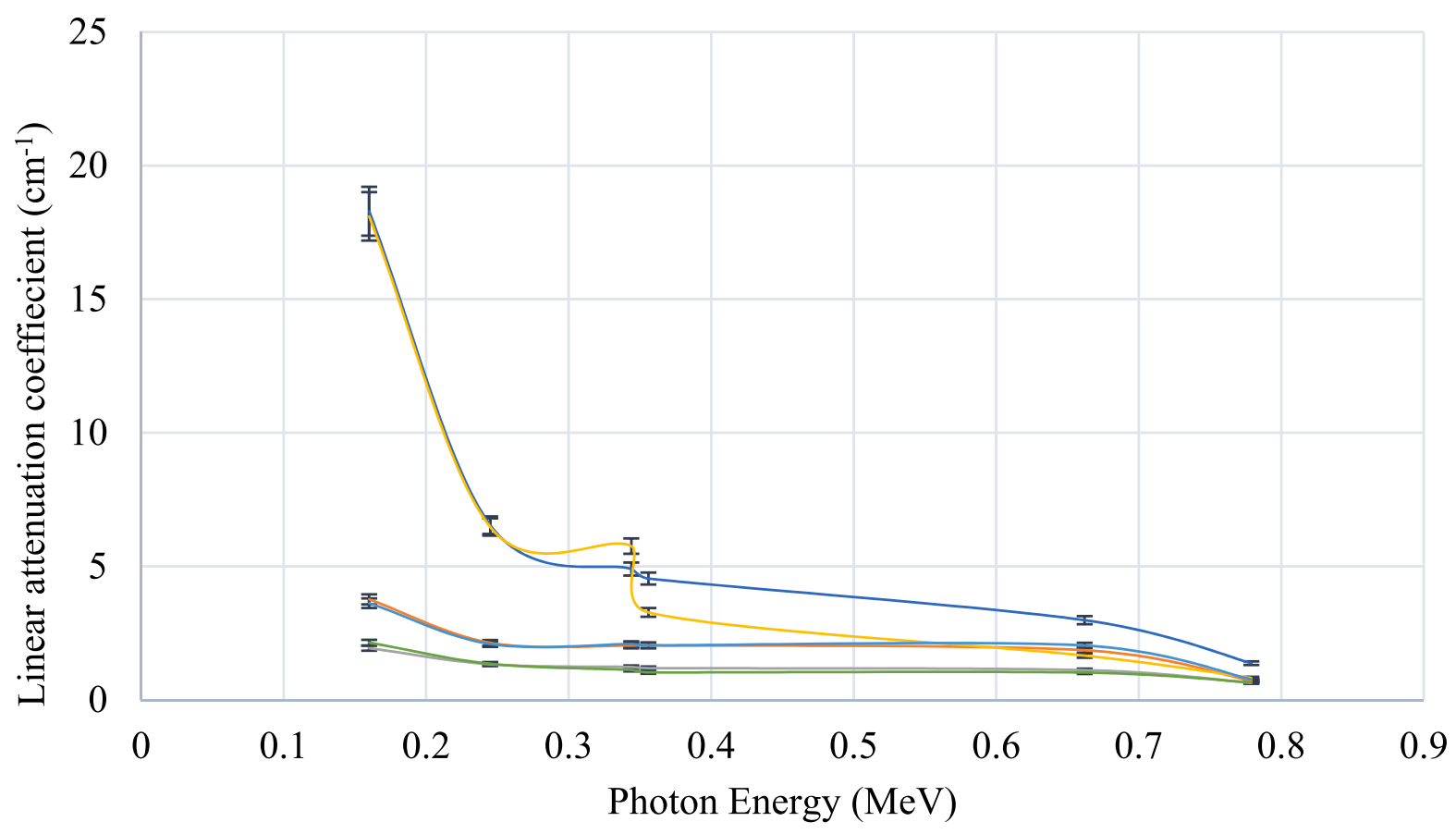

$-0.1 \mathrm{~cm} \mathrm{wc}-0.5 \mathrm{~cm} \mathrm{wc}-1 \mathrm{~cm} \mathrm{wc}-0.1 \mathrm{~cm} \mathrm{~Pb}-0.5 \mathrm{~cm} \mathrm{~Pb}-1 \mathrm{~cm} \mathrm{~Pb}$

Fig. 9. The linear attenuation coefficients of tungsten carbide (WC) and lead $(\mathrm{Pb})$ at different energies.

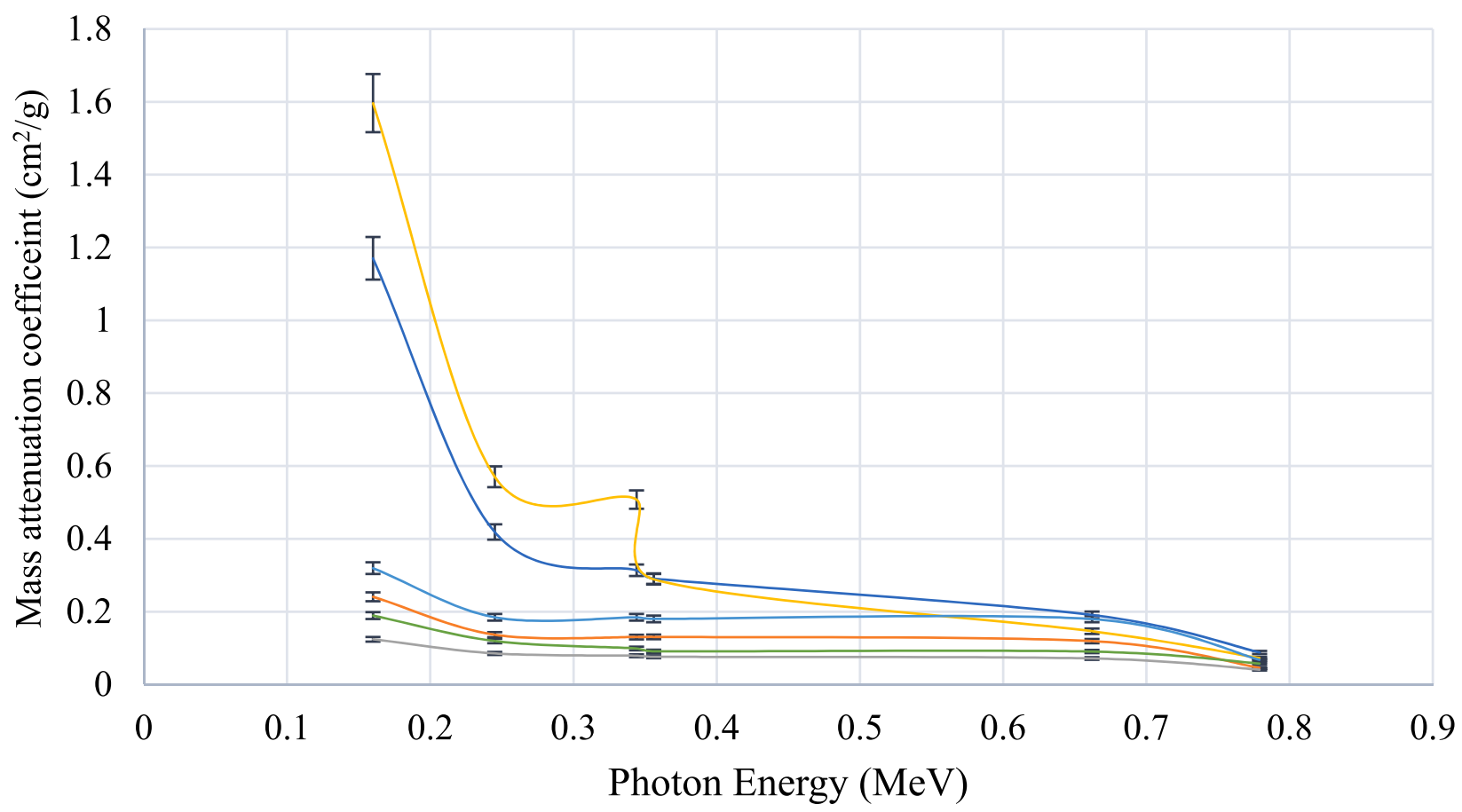

$-0.1 \mathrm{~cm} \mathrm{wc}-0.5 \mathrm{~cm} \mathrm{wc}-1 \mathrm{~cm} \mathrm{wc}-0.1 \mathrm{~cm} \mathrm{~Pb}-0.5 \mathrm{~cm} \mathrm{~Pb}-1 \mathrm{~cm} \mathrm{~Pb}$

Fig. 10. The mass attenuation coefficients of tungsten carbide (WC) and lead $(\mathrm{Pb})$ at different energies.

showed that the $P$-value obtained from this study for both tungsten carbide and lead were higher than 0.05 . It indicates that the mass attenuation coefficients of both materials have no significant difference.

In addition, the half value layer and mean free path demonstrate the radiation shielding material performance. Low half value layer and mean free path of material gives better radiation shielding performance because small volume of material is required for radiation shielding. This study found that tungsten carbide show better radiation shielding performance compared to lead at all energies. Small thickness of tungsten carbide is required for radiation shielding compared to lead at 


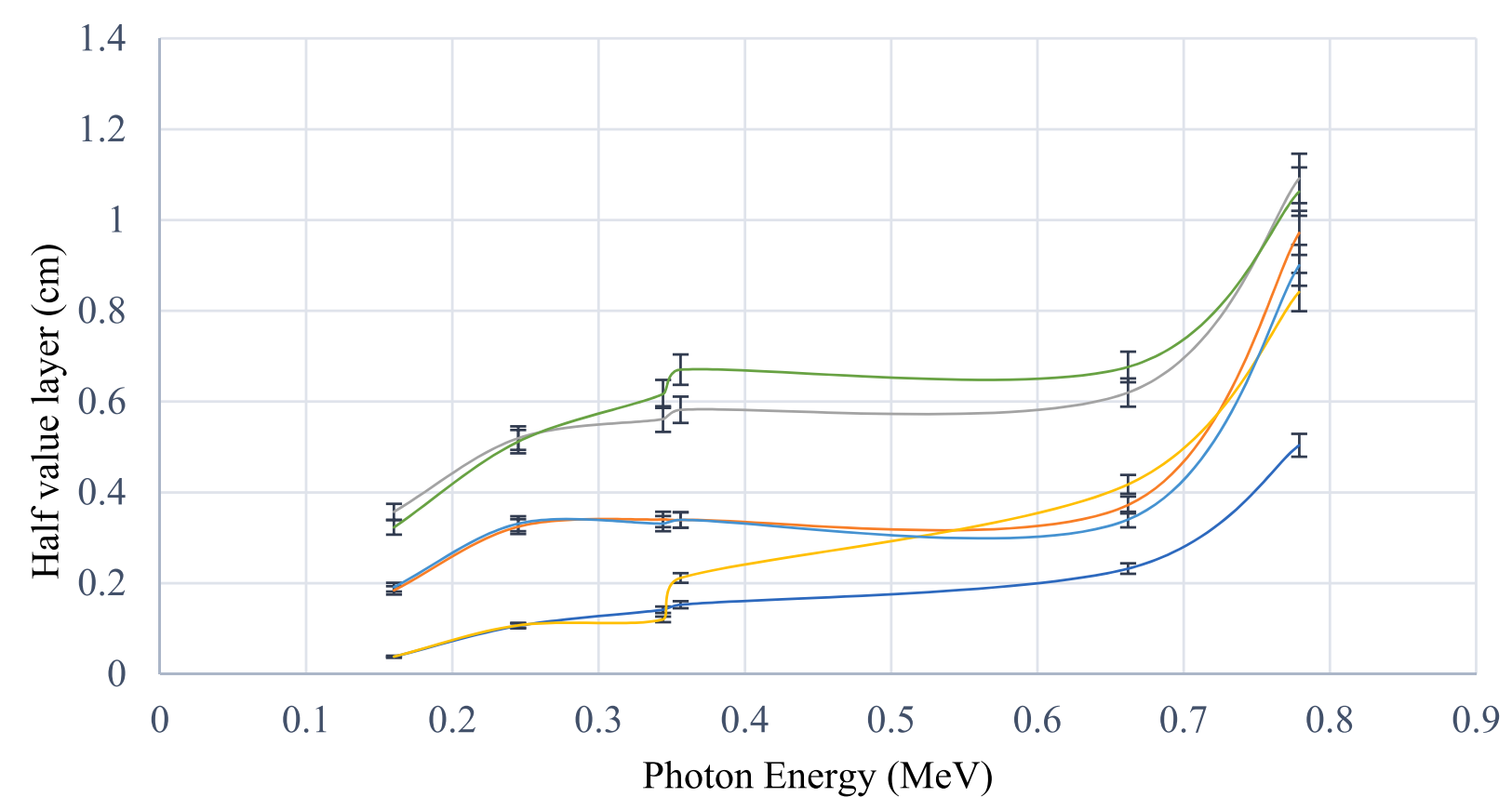

$-0.1 \mathrm{~cm} \mathrm{wc}-0.5 \mathrm{~cm} \mathrm{wc}-1 \mathrm{~cm} \mathrm{wc}-0.1 \mathrm{~cm} \mathrm{~Pb}-0.5 \mathrm{~cm} \mathrm{~Pb}-1 \mathrm{~cm} \mathrm{~Pb}$

Fig. 11. The half value layer of tungsten carbide $(\mathrm{WC})$ and lead $(\mathrm{Pb})$ at different energies.

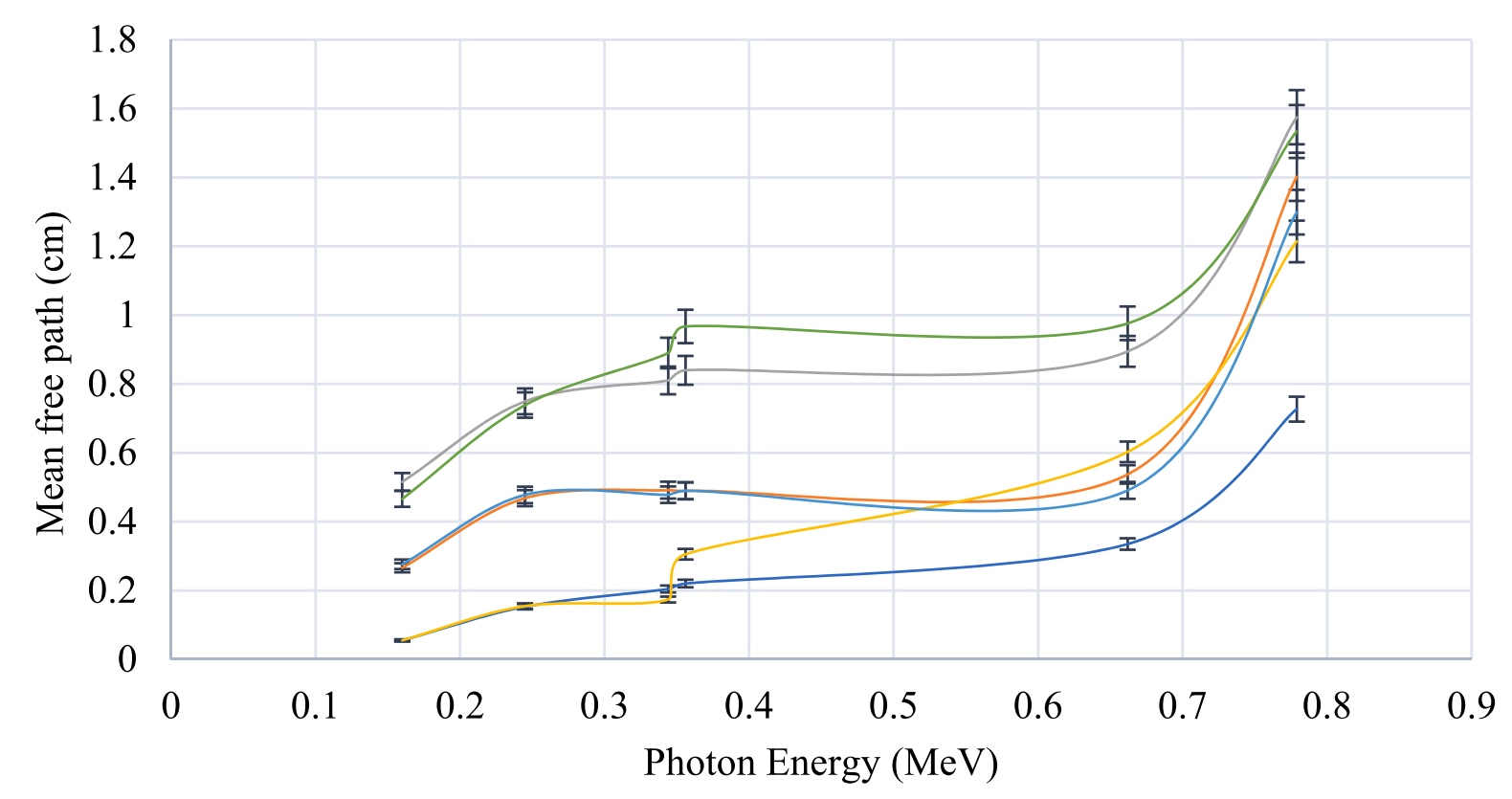

$-0.1 \mathrm{~cm} \mathrm{wc}-0.5 \mathrm{~cm} \mathrm{wc}-1 \mathrm{~cm} \mathrm{wc} \longrightarrow 0.1 \mathrm{~cm} \mathrm{~Pb}-0.5 \mathrm{~cm} \mathrm{~Pb}-1 \mathrm{~cm} \mathrm{~Pb}$

Fig. 12. The mean free path of tungsten carbide (WC) and lead (Pb) at different energies.

same energy. The radiation shielding performance of tungsten carbide in terms of half value layer and mean free path analysis show that tungsten carbide performance is consistent across the energies compared to lead. This study demonstrates that tungsten carbide has a potential to replace lead in radiation shielding for nuclear medicine application. Studies done by Kobayashi et al., [16] and Division [17] also highlighted the ability of pure tungsten and tungsten carbide-cobalt in replacing lead for radiation protection.
This study show that tungsten carbide can be used to substitute lead as radiation shielding material in nuclear medicine. This study also found that tungsten carbide attenuated more radiation compared to pure tungsten, tungsten carbide cobalt and tungsten reinforced basaltfiber. Therefore, it proves that pure tungsten carbide is a good lead-free radiation shielding material for nuclear medicine application. 
Table 5

The mass attenuation coefficient of tungsten carbide and lead at $0.160 \mathrm{MeV}-0.779 \mathrm{MeV}$.

\begin{tabular}{|c|c|c|c|c|c|c|}
\hline \multirow[t]{2}{*}{ Energy (MeV) } & \multicolumn{3}{|l|}{ Lead } & \multicolumn{3}{|c|}{ Tungsten Carbide } \\
\hline & Experiment & Theoretical & $\Delta(\mu / \rho)(\%)$ & Experiment & Theoretical & $\Delta(\mu / \rho)(\%)$ \\
\hline 0.160 & 1.602 & 1.626 & 1.48 & 1.207 & 1.272 & 5.11 \\
\hline 0.245 & 0.552 & 0.580 & 4.98 & 0.469 & 0.471 & 0.42 \\
\hline 0.344 & 0.272 & 0.283 & 4.09 & 0.239 & 0.240 & 0.67 \\
\hline 0.356 & 0.261 & 0.265 & 1.73 & 0.209 & 0.209 & 0.19 \\
\hline 0.662 & 0.1291 & 0.130 & 1.07 & 0.090 & 0.091 & 1.19 \\
\hline 0.779 & 0.0824 & 0.086 & 4.65 & 0.077 & 0.078 & 0.84 \\
\hline
\end{tabular}

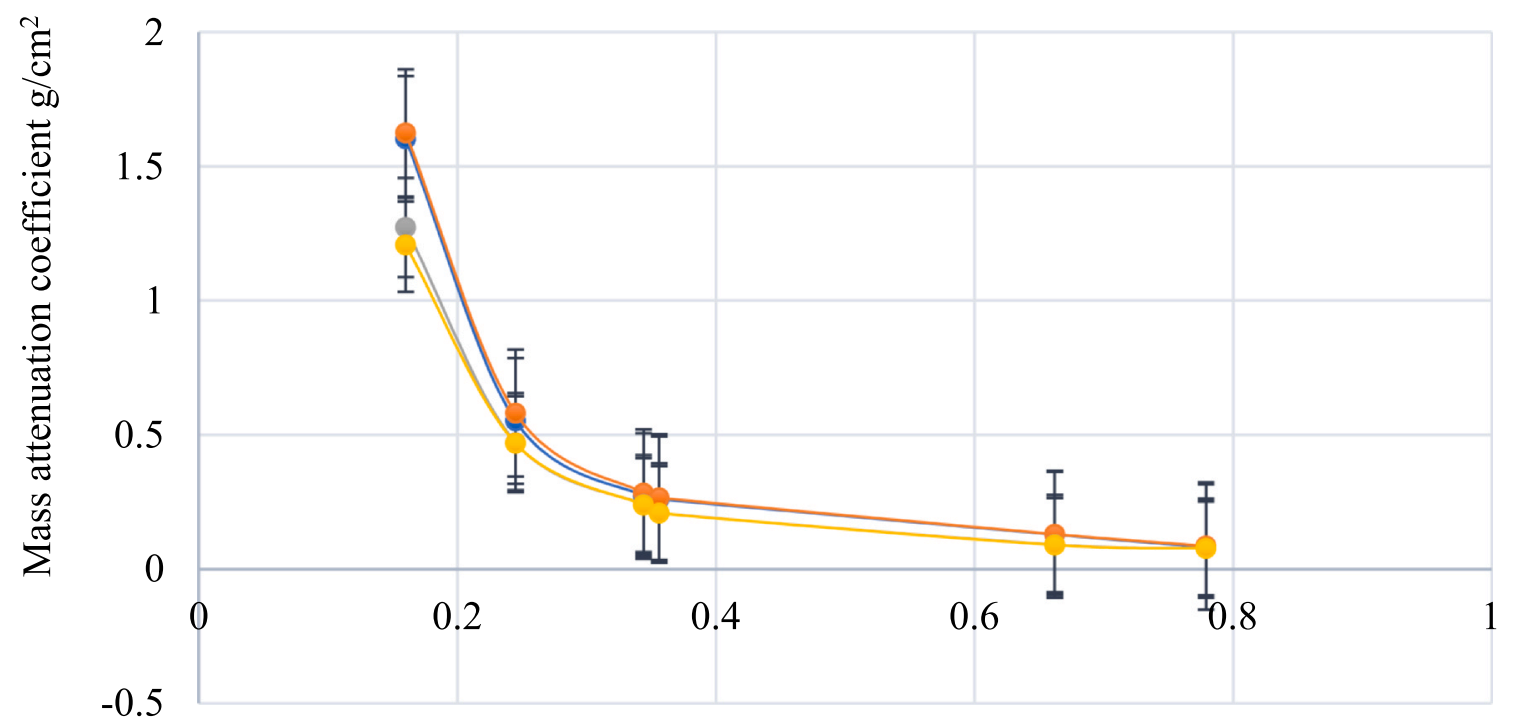

\section{Photon Energy (MeV)}

\section{-lead (Experimental) $\longrightarrow-$ lead $(\mathrm{XCOM}) \longrightarrow$ WC (XCOM) $\rightarrow-$ WC (Experimental)}

Fig. 13. The measured and theoretical mass attenuation coefficients of tungsten carbide and lead at different energy.

Table 6

The mass attenuation coefficient of tungsten carbide at $0.662 \mathrm{MeV}$ obtained from this study compared to other previous works.

\begin{tabular}{lll}
\hline Study & $\begin{array}{l}\text { Radiation shielding } \\
\text { material }\end{array}$ & $\begin{array}{l}\text { Mass attenuation coefficient at } \\
0.662 \mathrm{MeV}\end{array}$ \\
\hline This study & Tungsten carbide & 0.09 \\
B. Buyuk [17] & $\begin{array}{l}\text { Tungsten } \\
\text { Tungsten carbide- }\end{array}$ & 0.08 \\
& Cobalt & 0.08 \\
& Lead & 0.09 \\
Yingwei Hou [26] & Tungsten-Fiber & 0.08 \\
\hline
\end{tabular}

\section{Conclusion}

In conclusion, this study showed that tungsten carbide has a potential to replace lead as a new lead-free radiation shielding material in nuclear medicine. It can be used as a radiation shielding material to stop the gamma radiation. The use of lead-free radiation shielding material is important to minimise toxic impact to our environment and health.

\section{Declaration of Competing Interest}

The authors declare that they have no known competing financial interests or personal relationships that could have appeared to influence the work reported in this paper.

\section{Acknowledgment}

The authors would like to thank nuclear medicine technologist and laboratory assistant at Nuclear Medicine Unit in Advanced Medical and Dental Institute, Universiti Sains (USM) and Biophysics Laboratory, School of Physics, USM respectively for the technical assistance in this study. The authors also would like to acknowledge financial support from USM for the Bridging Grant (304/CIPPT/6316267), Graduate on Time Incentive (1001/CIPPT/823194) and USM Fellowship for this study.

\section{References}

[1] Obaid SS, Gaikwad DK, Pawar PP. Determination of gamma ray shielding parameters of rocks and concrete. Radiat. Phys. Chem. 2017.

[2] Manjunatha HC, Seenappa L, Chandrika BM, Hanumantharayappa C. Annals of nuclear energy a study of photon interaction parameters in barium compounds. Ann. Nucl. Energy 2017;109:310-7.

[3] Singh M, Tondon A, Sandhu BS, Singh B. Energy dependence of radiation interaction parameters of some organic compounds. Radiat. Phys. Chem. 2017.

[4] Obaid SS, Sayyed MI, Gaikwad DK, Pawar PP. Attenuation coefficients and exposure buildup factor of some rocks for gamma ray shielding applications. Radiat. Phys. Chem. 2018.

[5] R. Mirji and B. Lobo, "Radiation shielding materials : A brief review on methods, scope and significance," Natl. Conf. Adv. VLS Microelectron. 27th Januari 2017, P. C. Jabin Sci. Collecge, Huballi, India, no. June, 2017.

[6] Kaur T, Sharma J, Singh T. Progress in Nuclear Energy Review on scope of metallic alloys in gamma rays shield designing. Prog. Nucl. Energy 2019;113:95-113.

[7] Wani AL, Ara A, Usmani JA. Lead toxicity : a review. Interdiscip. Toxicol. 2015;8(2):55-64.

[8] Bushberg JT, Seibert JA, Boone JM. The Essential Physics of Medical Imaging 
EDITION. Wolters Kluwer: Thired Edi; 2012.

[9] D. R. Mcalister and D. Ph, "Gamma Ray Attenuation Properties of Common Shielding Materials," 2013.

[10] Oxide T, Okuyama F. Tungsten nanoparticles influence on radiation protection properties of polymers Tungsten nanoparticles influence on radiation protection properties of polymers. IOP Conf. Ser Mater. Sci. Eng. 2016.

[11] C. Rudolph, A. Rudolph, G. Lister, L. First, and A. Gershon, Rudolph's Pediatric, 22nd ed. 2011.

[12] N. J. Abualroos, N. A. Baharul Amin, and R. Zainon, "Conventional and new leadfree radiation shielding materials for radiation protection in nuclear medicine : A review," Radiat. Phys. Chem., vol. 165, no. December, 2019.

[13] R. Release, "Environment : Fewer risks from hazardous substances in electrical and electronic equipment," no. July, 2011.

[14] European Parliament, "Directive 2011/65/EU of the European Parliment and of the council on the Restriction of the Use of Certain Hazardous Substances in Electrical and Electronic Equipment (RoHS) - recast," Off. J. Eur. Union, pp. 88-110, 2011.

[15] T. Union, O. Journal, and E. Union, "(Text with EEA relevance)," pp. 1-38, 2019.

[16] S. Kobayashi, N. Hosodaa, and R. Takashimab, "Tungsten alloys as radiation protection materials," vol. 390, pp. 426-430, 1997.

[17] N. R. Division, "Comparison of Lead and WC-Co Materials against Gamma Irradiation,” vol. 125, no. 2, pp. 423-426, 2014.

[18] Luković J, et al. Synthesis and characterization of tungsten carbide fine powders. Ceram. Int. 2015;41(1):1271-7.
[19] Selyutin GE, Gavrilov YUYU, Voskresenskaya EN, Zakharov VA, Nikitin VE, Poluboyarov VA. Composite materials based on ultra high molecular polyethylene: properties. Appl. Prospects 2010;18:301-14.

[20] S. D. Kaloshkin, V. V Tcherdyntsev, M. V Gorshenkov, V. N. Gulbin, and S. A. Kuznetsov, "Radiation-protective polymer-matrix nanostructured composites," vol. 3, pp. 522-526, 2012.

[21] Chang L, et al. Preparation and characterization of tungsten/epoxy composites for $\gamma$-rays radiation shielding. Nucl. Instrum. Methods Phys. Res. Sect. B Beam Interact. Mater. Atoms 2015;356(8):88-93.

[22] Lukovi J, Babi B, Prekajski M, Panti J. Synthesis and characterization of tungsten carbide fine powders. Ceram. Int. 2014.

[23] Singh K, Rani A, Singh M. Shielding behaviors of some polymer and plastic materials for gamma-rays. Radiat. Phys. Chem. 2015;106(1):247-54.

[24] Islam M, Martinez-duarte R. A sustainable approach for tungsten carbide synthesis using renewable biopolymers. Ceram. Int. 2017.

[25] Soylu HM, Yurt Lambrecht F, Ersöz OA. Gamma radiation shielding efficiency of a new lead-free composite material. J. Radioanal. Nucl. Chem. 2015;305(2):529-34.

[26] Y. Hou, M. Li, Y. Gu, Z. Yang, R. Li, and Z. Zhang, "Gamma Ray Shielding Property of Tungsten Powder Modified Continuous Basalt Fiber Reinforced Epoxy Matrix Composites," pp. 1-10, 2018.

[27] Berger MJ, Hubbell JH, Seltzer SM, Chang J, Coursey JS, Sukumar R, et al. January 8]. Gaithersburg, MD: National Institute of Standards and Technology; 2020. 